\title{
ULTRASONIC TECHNOLOGICAL MACHINE MODELS
}

\author{
Vladimir Astashev ${ }^{1}$, Vitaly Krupenin ${ }^{1,2}$ \\ ${ }^{1}$ Mechanical Engineering Research Institute of the Russian Academy of Sciences, Russia; \\ ${ }^{2}$ Moscow Polytechnic University, Russia \\ v_astashev@mail.ru, krupeninster@gmail.com
}

\begin{abstract}
The article discusses the problems of dynamic modeling of ultrasonic technological machines and devices. These systems are widely used in modern agricultural and industrial production, in many branches of modern mechanical and instrument engineering, as well as in medical industry due to specific and unique properties of various types of ultrasonic treatment. Among these properties, one should mention, efficacious effect on hard and brittle materials; formation of surface hardening of processed materials, increase in plasticity of materials in the ultrasonic field, nanostructuring of surface and near-surface layers of parts during processing, and many others. For this reason, ultrasonic methods are applied extensively and the development of dynamic modeling schemes for the corresponding technical means appears to be very important. The components of the model of any machine: "the engine - the mechanical system - the working process - the control system" for ultrasonic devices acquires its specific and very peculiar character, determined, in particularly, by the high frequency of the technological process. The exciter of the ultrasonic oscillations, piezoelectric or magnetostrictive one, acts now as the engine. The function of the mechanical system that transmits vibrations from the exciter to the working tool is performed by the waveguide. The vibroexciter is powered by the generator of electrical oscillations. The control systems are designed to maintain the necessary vibration settings of the working tool and contain feedback subsystems. These systems are largely responsible for the efficiency of the ultrasonic technological process and determine the expediency of its application. A general approach to the models of various ultrasonic technological systems is developed in this article.
\end{abstract}

Keywords: ultrasonic technology, machine model, oscillatory system, operator equations of motion.

\section{Introduction}

Ultrasonic technological machines belong to the class of vibrating machines [1;2], however, they are ranked among a separate group for the following main reasons.

The first of these is determined by the main features of the behavior of materials and media in the ultrasonic field that have been identified in numerous experiments. These features are manifested through a radical change in their elastoplastic characteristics and rheological properties, such as brittleness, ductility and viscosity.

For example, dry friction in the contact zone of two surfaces under the influence of ultrasonic vibration transforms into viscous friction [3; 4]. When the samples are subject to deformation, with the imposition of ultrasonic vibration, one can observe a significant reduction in the yield strength of the materials.

The second reason is due to the specific design of the main elements of the machine, which are rod oscillatory systems, formed, as a rule, by inhomogeneous sections, and operating in waveguide mode [3; 4]. For this reason, to describe the oscillations, the basic elements of the machine are modeled by systems with distributed parameters and are described by partial differential equations.

The interaction of the working body with the workpieceresults in a strong nonlinearity of the oscillatory system in operating modes.

The universal trine, which defines any vibrating machine - "the engine-oscillatory system technological process", in the case of ultrasonic machines is determined by the trine "ultrasonic vibration exciter-waveguide-ultrasonic process".

The working process of the ultrasonic technological machine is carried out by the working body, which in addition to the forming motion relative to the workpiece or medium undergoes highfrequency (ultrasonic) oscillations of a certain direction, frequency, and intensity. The design of the machine and its elements is largely determined by the scheme of the technological process performed by the working body.

The oscillations are excited by the motor, which in most cases is a magnetostrictive or piezoelectric vibrational exciter. Then the vibrations are transferred to the working medium by an oscillating system (waveguide); often this happens with a change in direction and amplitude. 
Because of the highly nonlinear dynamic properties of modern ultrasonic technological machines and also because of the high frequency of the actual vibration, they are usually equipped with very specific control systems [3].

\section{Technological process schemes}

Let us consider some typical ultrasonic technological processes (Fig.1). Fig. 1, a shows a possible scheme for cutting brittle and hard materials.
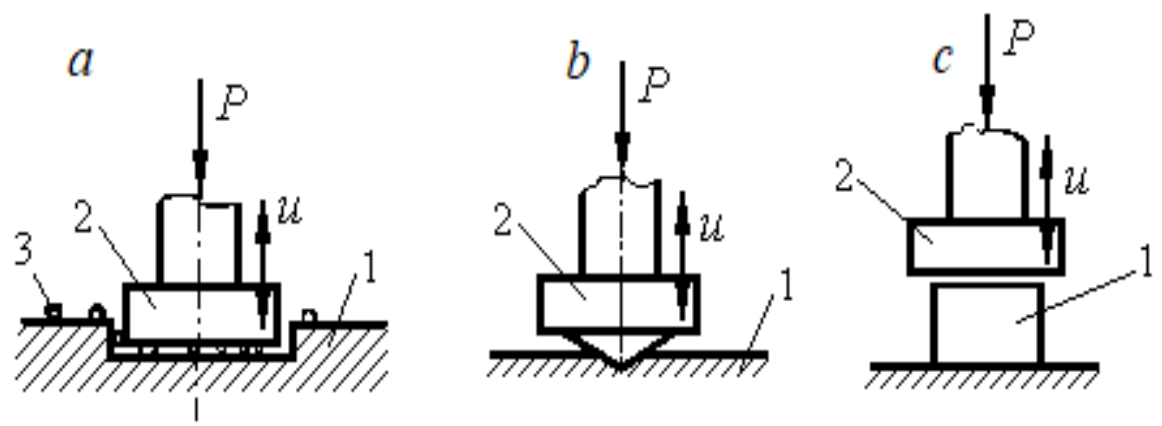

Fig. 1. Ultrasonic treatment: $a$ - cutting; $b$ - surface hardening; $c$-metal sediment

The blank 1 is located below the surface of the tool 2, which oscillates at a high frequency in a direction perpendicular to the treatment surface. The abrasive slurry is fed into the working zone formed by the tool surface and the surface of the workpiece. The tool is pressed against the product by means of a static feed force $P$. The tool acts on the workpiece material through abrasive grains that are deposited from the slurry. The surface of the material is thus damaged by creating a recess that copies the shape of the tool.

After its invention [5], the ultrasonic treatment method has been recognized and widely used for cutting materials that are difficult to apply to traditional methods such as glass, ceramics, semiconductor materials, ferrite, diamond, hard alloys, etc. Numerous experiments $[3 ; 6]$ showed that the destruction of the material occurs solely because of the tool interacting with the treated surface through abrasive particles. It is shown that the movement of the tool is a periodic vibro-impact process, in which collisions with the workpiece occur with the tool's oscillation frequency, while the amplitude of the impact force is the main factor affecting the cutting speed [7].

The cutting speed, other things being equal, increases with increasing amplitude and frequency of the tool vibrations, as well as the static feed force [7-9]. Brittle materials are better machineable; the cutting speed being the higher, the lower the hardness of the material.

To destroy the material between the tool and the workpiece, it is necessary to create, near the abrasive grains, forces sufficient to produce stresses that exceed the strength limit of the material being machined. These forces can reach significant values. At the same time, cutting takes place even at very low efforts of static pressing the tool to the workpiece.

Thus, ultrasonic vibrations in the treatment zone allow a significant decrease for the static forces, necessary to overcome the limiting loads.

The phenomenon of reducing the necessary static forces is a characteristic feature of most ultrasonic technological processes associated with the destruction or plastic deformation of the medium being treated.Figure $1, \mathrm{~b}$ shows the scheme of the process of surface hardening $[10 ; 11]$ of the workpiece 1, caused by the plastic deformation of the surface layer of the metal with ultrasonic vibrations of the tip 2 . In this case, a relatively small static pressing force $P$ is required to maintain the technological process, which is usually an order of magnitude less than its value, necessary for plastic deformation in the absence of oscillation of the tip.

Ultrasonic surface hardening proceeds most intensively and the reduction of the static clamp is most significant, when the operating mode of tip oscillations is a periodic vibro-impact process [12], as in the case of ultrasonic cutting (Fig. 1, a).

It should also be noted, that hardening is accompanied by occurrence of "correct" nanostructure formations in the surface and near-surface layers of the material [4]. 
A similar process is used in precipitation of metals (Fig. 1, c) with superposition of ultrasonic vibrations [13]. The sample 1 is subjected to joint action of static load $P$ and high-frequency oscillations of the tool 2. The action results in its plastic deformation and under the ultrasonic vibration the sample softens.

The list of popular ultrasonic technologies can be significantly extended: different types of welding, wire drawing, echolocation, flaw detection, etc. [10-14].

\section{Mathematical model of ultrasonic technological machine}

As noted above, in an ultrasonic technological machine, regardless of its purpose and design features, it is possible to indicate a high-frequency vibration excitation mechanism and an oscillating system serving to transfer vibration to the object being processed.

Proceeding from this, let us imagine the machine in the form of a generalized scheme, shown in Fig. 2, a. Here, the oscillatory system 2 is connected at the point 0 with the generator 1 , and at the point 1 interacts with the technological load 3 , which is created during the execution of the working process. The generator causes the transformation of electrical oscillations at its input into mechanical oscillations at the output 0 , which, in turn, is the input element of the oscillatory system. The oscillatory system transforms the input effects, creating the necessary conditions for the organization of the technological process at the output.

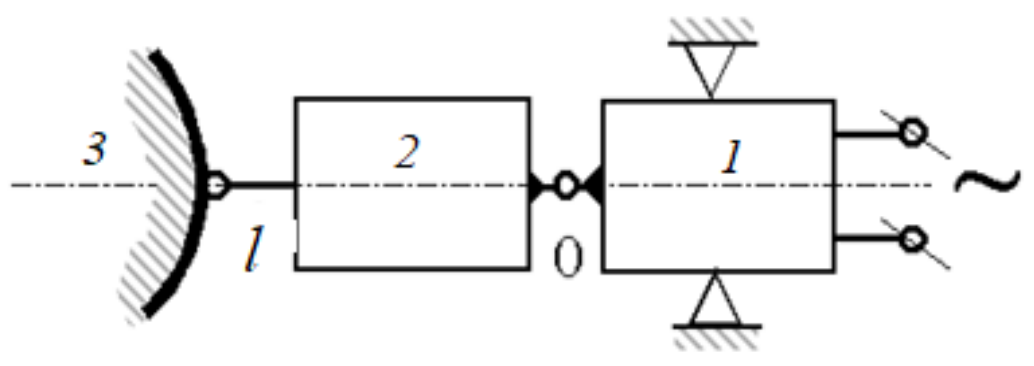

Fig. 2. Ultrasonic engine: 1 - generator; 2 - oscillatory system; 3 - technological load

Let us divide the received chain system into component subsystems, allocating the generator 1 , oscillating system 2 and technological load 3 and after that replacing their interaction by forces applied to the nodes. The forces acting on neighboring subsystems at the node point are equal in magnitude and opposite in direction. They differ only in sign. Now it is possible, considering first the individual parts of the system and composing the equations of motion of the mechanical (vibrational) subsystem and the electromechanical (exciter of oscillations) subsystem, to obtain a general equation of motion of the ultrasonic technological machine.

Specificity of the technological process can be considered using its dynamic characteristics [3;4].

It is convenient to write the equations in the operator form. In this case, we will use dynamic compliances [15], which connect forces applied to certain characteristic points of systems, with displacements at some other or the same points. The methods of dynamic compliance assume that the systems are linear.

Operators of dynamic compliance can be constructed not only based on differential or integrodifferential equations of motion, but also from the results of field experiments. Dynamic compliance is the response of linear systems to standard sinusoidal influences exp $(i \omega t)$.

It should also be noted that the operator methods are very useful for obtaining equations of electromechanical systems.

Denoting the dynamic compliance $L_{s x}(i \omega)$ to describe the steady-state oscillations of an arbitrary element $x$ of the oscillatory system, we write:

$$
u_{x}(t)=L_{0 x}(i \omega) \cdot f_{0}(t)-L_{l x}(i \omega) \cdot f_{l}(t),
$$

where $u_{x}(t)$ - a desired displacement;

$f_{0}(t)$ and $f_{l}(t)$ - forces, applied to elements " 0 " and " $l$ " (Fig.2);

$L_{0 x}(i \omega)$ and $L_{l x}(i \omega)$ - assigning the displacement of the element $x$ to these forces. 
Note that by symbol $(i \omega)$ we mean the operator of differentiation with respect to time.

Input element 1 (Fig. 2) is in fact a converter of the electric signal of electric current sources or voltage to ultrasonic vibration. For the output force, one can obtain a formula analogous to the operator expression (1), depending on the coefficients of the electromechanical coupling and determining the mutual influence of the electrical and mechanical parts of the drive, as well as other determining parameters.

A few transformations can lead to an operator equation for the technological machine in the form

$$
u_{l}(t)=u_{l 0}(t)-L_{M}(i \omega) \cdot f_{l}\left[u_{l}(t) ; \dot{u}_{l}(t)\right] ;
$$

where $u_{l}(t)$ - the law of motion of the working body of the machine;

$u_{l 0}(t)$ - the law of motion when idling;

$f_{l}\left[u_{l}(t) ; \dot{u}_{l}(t)\right]$ - dynamic characteristics of the working process (see below). motor

Complete, reduced to the working element, dynamic compliance of the oscillatory system and

$$
L_{M}(i \omega)=L_{l l}(i \omega)-\frac{L_{0 l}^{2}(i \omega)}{L_{00}(i \omega)+L_{n}(i \omega)},
$$

where $L_{n}(i \omega)$ - the dynamic compliance of the converter (Fig. 2, subsystem 1), which depends on the coefficients of electromechanical coupling and the type of the external power supply of the machine.

The dynamic characteristic of the working process in some cases can be represented by the linear dependence of the force $f_{l}$ on the displacement $u_{l}(t)$ of the working body $f_{l}(t)=L_{H}{ }^{-1}(i \omega) \cdot u_{l}(t)$, where $L_{H}(i \omega)$ is the dynamic compliance of the process load.

In this way one can describe, for example, the interaction of the tool with the workpiece during continuous deformation of the sample, occurring within the elastic zone of the material characteristic.

For linear elastic-viscous material, considering the type of the processes under consideration, we can write

$$
f_{l}(t)=k_{0} u_{l}(t)+\beta_{0} \dot{u}_{l}(t)=\left(k_{0}+i \omega \beta_{0}\right) u_{l}(t),
$$

where $k_{0}$ and $\beta_{0}$ - static rigidity of the sample and the coefficient of resistance to deformation.

In the general case, the characteristic $f_{l}$ is nonlinear. Some examples [4] of functions $f_{l}$ are shown in Fig. 3 and Fig. 4.

$a$

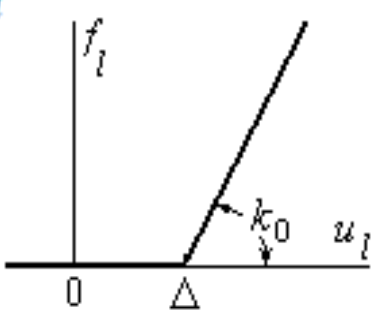

$b$

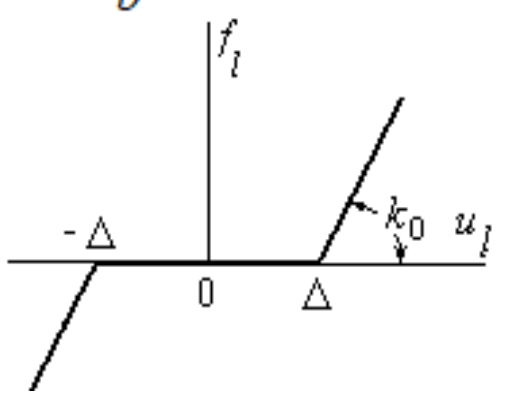

$c$

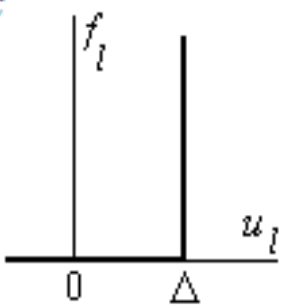

Fig.3. Dynamic characteristics of workflow

When the elastic specimen is deformed by a vibrating tool, it is possible to establish a periodic pulsed (vibro-impact) loading regime. Such process can be described by the characteristic of one-sided elastic limiter (Fig. 3, a), the equation of which has the form $f_{l}\left(u_{l}\right)=k_{0}\left(u_{l}-\Delta\right) \eta\left(u_{l}-\Delta\right)$, where $k_{0}$ is the static stiffness of the limiter; $\eta(u)$ is a unit function.

As a rule, $k_{0} \gg>1$ and therefore nonlinear characteristics of this type are called "strongly" [15]. Fig.3, $b$ shows a symmetric strongly nonlinear threshold characteristic. 
Providing the impact of the instrument may be considered as momentary, $k_{0} \rightarrow \infty$ and a stereomechanical impact model can be used. A symbolic view of the process characteristic is given in Fig. 3, c.

More realistic are the models that consider the energy loss during the interaction. When considering instantaneous collisions, a well-known Newton's hypothesis is used [17].

In cases where the shock interaction time cannot be neglected, the stereomechanical impact model is not applicable and other models must be used. One of them is shown in Fig. 4, a:

$$
f_{l}\left(u_{l}, \dot{u}_{l}\right)=k_{0}\left(u_{l}-\Delta\right) \eta\left(u_{l}-\Delta\right)\left(1+\theta \operatorname{sgn} \dot{u}_{l}\right) .
$$

The directions of deformation and unloading are determined by going around the hysteresis loop clockwise. The area of the loop determines the parameter $\theta$.

$a$

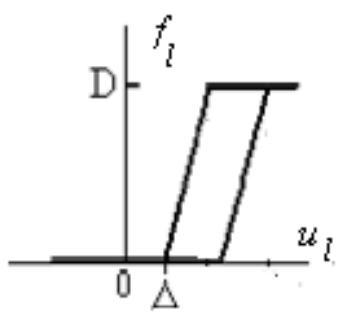

$b$

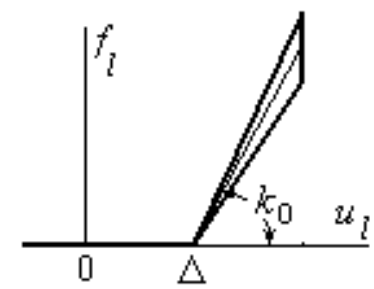

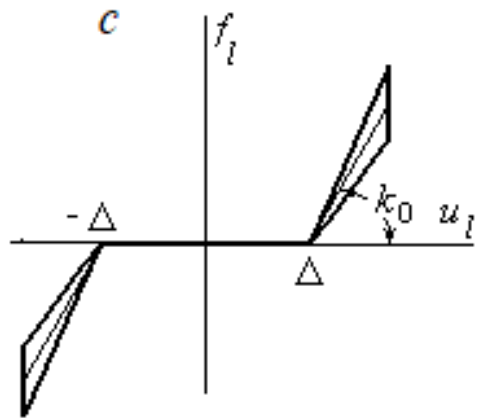

Fig. 4. Dynamic characteristics of workflow with hysteresis

Characteristics of this kind, in the general case, can be represented in the form

$$
f_{l}\left(u_{l}, \dot{u}_{l}\right)=f_{l}^{(1)}\left(u_{l}\right)+f_{l}^{(2)}\left(u_{l}, \dot{u}_{l}\right),
$$

where $f_{l}^{(1)}\left(u_{l}\right)$ determines the elastic forces;

$$
f_{l}^{(2)}\left(u_{l}, \dot{u}_{l}\right)=-f_{l}^{(2)}\left(u_{l},-\dot{u}_{l}\right) \text {, describes the dissipative forces. }
$$

Fig. $4, b$ demonstrates the symmetrical analog of the given characteristic

$$
f_{l}\left(u_{l}\right)=k_{0}\left(\left|u_{l}\right|-\Delta\right) \eta\left(\left|u_{l}\right|-\Delta\right) \operatorname{sgn} u_{l}\left(1+\theta \operatorname{sgn} \dot{u}_{l}\right) .
$$

Finally, Fig. 4, $c$ shows the dynamic characteristic of the plastic deformation of the material in the pulsed regime $[3 ; 4]$. The value $D$ is the limit of elasticity.

Note that the form of the operator equation (2) reveals the fact that tuning the machine to the maximum efficiency mode (resonance) should be performed at frequencies different from the resonance at idling, when the function $u_{l 0}(t)$ reaches its maximum. This circumstance is very important, since it requires special methods of analysis [3; 4].

We should also note that the numerous diverse applications of ultrasonic technologies $[18 ; 19]$ should not obscure their apparent "vibrational nature".

\section{Conclusions}

Ultrasonic technology provides a universal way of organizing the impact on workpieces and materials, remaining a subclass of vibrational technologies, and the main components of the determining elements of each vibrating machine "engine-oscillatory system-technological process" are defined as follows: "generator (electromechanical exciter)-waveguide-ultrasonic technological process". Ultrasonic technological machines can be considered as a subclass of vibro-impact machines and analyzed from a single point of view, and described by a nonlinear operator equation, to which the methods of modern nonlinear mechanics and the theory of oscillations and waves can be applied.

\section{Acknowledgements}

This work was supported by the Russian Foundation for Basic Research (project 18-08-00168). 


\section{References}

[1] Astashev V.K, Babitsky V.I., Kolovsky M.Z.Dynamics and Control of Machines. Berlin: Springer-Verlag., 2000. 235 p.

[2] Вибрации в технике: Справочник. Т.4. Вибрационные машины и процессы. (Vibrationin Engineering: Handbook. Vol.4. Vibration machines and processes.) Lavendelis E.E (ed.), Moscow:Mashinostroenie, 1981. 510 p. (In Russian).

[3] Astashev V.K., Babitsky V.I. Ultrasonic Processes and Machines. Dynamics, Control and Applications. Berlin: Springer-Verlag, 2007. 330 p.

[4] Асташев В.К., Крупенин В.Л. Нелинейная динамика ультразвуковых технологических процессов (Nonlinear Dynamics of Ultrasonic Technological Processes). Moscow: MPU(MGUP), 2016. 372 p.(In Russian).

[5] Farrer J.O. Method of Abrading. - Patent of Great Britain No 602801, 1948.

[6] Розенберг Л.Д., Казанцев В.Ф. О физике ультразвуковой обработки твердых материалов. (On the physics of ultrasonic treatment of solid materials.) Rep. Ac. Sci. USSR, 1959, vol. 124, No 1, pp. 79-82.(In Russian).

[7] Nishimura G., Shimakawa S. Ultrasonic mechanical machining (Part IV). - Journ. Fac. Eng. Univ. Tokyo, 1959, v. 26, No 1, pp. 13-35.

[8] Goetze D. Effect of Vibration Amplitude, Frequency and Composition of Abrasive Slurry on the Rate of Ultrasonic Machining in Ketoes Tool Steel. Journ. Acoust. Sos. Amer., 1956, vol. 28, No 6, pp. 1033-1037.

[9] Neppiras E.A. Report on ultrasonic machining. Metalworking production, 1956, vol. 100, No 29, pp. 1377-1382.

[10] Марков А.И. Ультразвуковая обработка материалов. (Ultrasonic processing of materials.) Moscow:Mashinostroenie, 1980. 237 p. (In Russian).

[11]Марков А.И., Озерова М.А., Устинов И.Д. Применение ультразвука при алмазном выглаживании деталей. (Application of ultrasound for diamond smoothing of parts). Bulletin of Machine Building, 1973, No 9, pp. 57-61. (In Russian).

[12] Astashev V.,Babitsky V. Ultrasonic cutting as a non-linear (vibro-impact) process. Ultrasonics, 1998, No 6, pp. 89-96.

[13] Северденко В.П., Клубович В.В., Степаненко А.В. Ультразвук и пластичность (Ultrasonic and plasticity). Minsk: Science and Technology, 1976. 446 p. (In Russian).

[14] Babitsky V.I., Krupenin V.L Vibration of Strongly Nonlinear Discontinuous Systems. Berlin: Springer-Verlag, 2001. 404 p.

[15] Лурье И.А. Крутильные колебания в дизельных установках. (Torsional vibrations in diesel engines). Moscow-Leningrad: Voenmorizdat, 1940. 219 p.

[16] Терских В.П. Метод цепных дробей в применении к исследованию колебаний механических систем. (The method of continued fractions as applied to the study of oscillations in mechanical systems). Leningrad: Sudpromgiz, 1955. 708 p.

[17]Пановко Я.Г. Введение в теорию механического удара. (Introduction to the theory of mechanical shock).Moscow: Nauka, 1977. 224 p. (In Russian).

[18] Baldev Raj, V.Rajendran, P PalanichamyScience and technology of ultrasonics. New Delhi: Narosa Publishing House, 2003. 480 p.

[19] Ensminger D., Leonard J. Bond Ultrasonics: Fundamentals, Technologies, and Applications. CRC Press, Taylor\&Francis Group, USA, 2011. 690 p. 